\title{
Optimization of the power supply system for connection of the end user to electric grids according to the scheme of the third category of reliability
}

\author{
Ivan Bandurin ${ }^{1, *}$, Alexey Khaimin ${ }^{1}$ \\ ${ }^{1}$ Pskov State University, Faculty of Computer Science and Power Engineering, 180000, Russia, Pskov, Lenin Square, 2
}

\begin{abstract}
The article first developed a mathematical model of optimization of the power supply system for connection of the end user to electric grids according to the scheme of the third category of reliability. The mathematical model allows to choose the optimal type and brand power system equipment.
\end{abstract}

\section{Introduction}

At present, the Russian Federation uses a normative approach to ensure reliability. In total there are 3 categories of reliability of electric receivers: I-st, II-th and III-th [4]. By the degree of importance of the category number of consumers increases from the III-th to I-st. Consumers, for whom it is important to ensure uninterrupted power supply, must be connected in accordance with scheme I of the category and, on the contrary, unrelated consumers are connected according to scheme III of the category. Note that the majority of end-users are now connected according to scheme III reliability category.

However, in the article Vorotnitskiy VV [1] for example, it is shown that the reliability of electricity supply to a consumer of category III can be higher than a consumer of category II. It follows that the power supply reliability category can no longer be an expression of that same reliability, so it is better to use the reliability indices. The main indicators of the reliability of power supply to the end user are:

- average number of outages per year;

- average time of restoration of power supply.

Thus, the reliability of electricity supply to consumers in market conditions becomes the subject of contractual relations between the subjects of the energy market and the goods with the corresponding price realized through market services. In this regard, the urgency of the problem of choosing rational schemes of power supply to ensure the contractual reliability of endusers of electricity is growing [1-3].

The article considers a mathematical model for the selection of a rational power supply system for connection of the end user to electric grids according to scheme III of reliability category. The power supply system must provide a certain level of reliability of electricity supply. A review of publications [3, 5-10] showed that in such a direction the problem is solved for the first time.

\section{Mathematical model}

A schematic diagram of the power supply system for connecting the consumer to electrical networks is shown in Fig. 1. We will compile a mathematical model for choosing a rational system of electricity supply. For this we introduce the initial data, the objective function, constraints objective function.

a)

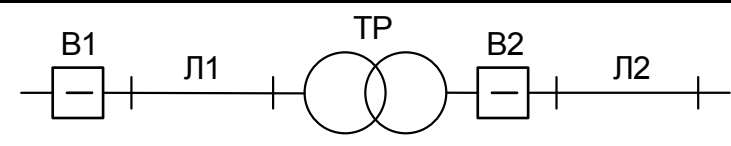

б)

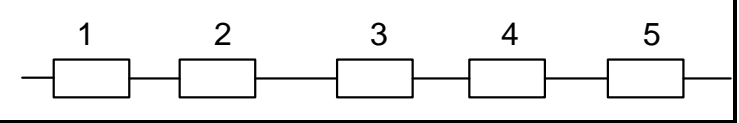

Fig. 1 - Schematic diagram of power supply (a) and the structural diagram of reliability of the consumer of the III reliability category (b), where 1, 4 - circuit-breaker, 2 - high voltage power line section, 3 - power transformer, 5 - low voltage power line section

Initial data (the input variables of the model): $L$ - distance from the nearest power center to the consumer, $\mathrm{km}$;

$P_{\text {max }}$ - maximum power of the consumer, $\mathrm{kW}$;

$U_{n}$ - consumer nominal voltage, $\mathrm{kV}$;

$\cos \varphi$ - power factor of the consumer;

$N$ - average number of outages, outages/year;

$T$ - average time of restoration of power supply, h;

$\Delta U$ - voltage loss in the power supply system, \%;

$\Delta P$ - power loss in the power supply system, $\%$.

\subsection{Variables of the mathematical model}

1 High and low voltage line type:

Corresponding author: bandurin ivan@mail.ru 
$y_{U W l i}=\left\{\begin{array}{l}1, \text { if the line is made of uninsulated wire, } \\ 0, \text { otherwise. }\end{array} ;\right.$

$y_{\text {SIWli }}=\left\{\begin{array}{l}1, \text { if the line is made of self-supporting insulated wire (SIW), } \\ 0, \text { otherwise. }\end{array}\right.$

$y_{C L l i}-\left\{\begin{array}{l}1, \text { if the line is cable, } \\ 0, \text { otherwise. }\end{array}\right.$

2 Length of high and low voltage line:

$L_{U W l i}$ - length of a line of uninsulated wire, $\mathrm{km}$;

$L_{S I W l i}$ - length of the line made by SIW, km;

$L_{C L l i}$ - length of the line made by the cable, $\mathrm{km}$.

3 Cross-section of high and low voltage line:

$F_{U W l i}$ - cross-section of a line of uninsulated wire, $\mathrm{mm}^{2}$;

$F_{S I W l i}$ - cross-section of the line of the SIP, $\mathrm{mm}^{2}$;

$F_{C L l i}$ - cross-section of the line made by cable, $\mathrm{mm}^{2}$.

4 Type of power transformer:

$y_{\text {OILtr }}-\left\{\begin{array}{l}1, \text { if the transformer is oily, } \\ 0, \text { otherwise. }\end{array} ;\right.$

$y_{\text {DRYtr }}-\left\{\begin{array}{l}1, \text { if the transformer is dry, } \\ 0, \text { otherwise. }\end{array} ;\right.$

$y_{S F 6 t r}-\left\{\begin{array}{l}1, \text { if gas-insulated transformer, } \\ 0, \text { otherwise. }\end{array}\right.$

5 Type of high and low voltage circuit-breaker:

$y_{A I R b i}-\left\{\begin{array}{l}1, \text { if the breaker is airy, } \\ 0, \text { otherwise. }\end{array}\right.$;

$y_{V A C b i}-\left\{\begin{array}{l}1, \text { if the breaker is vacuum, } \\ 0, \text { otherwise. }\end{array} ;\right.$

$y_{O I L b i}-\left\{\begin{array}{l}1, \text { if the breaker is oily, } \\ 0, \text { otherwise. }\end{array} ;\right.$

$y_{E M b i}-\left\{\begin{array}{l}1, \text { if the breaker is electromagnetic, } \\ 0, \text { otherwise. }\end{array}\right.$

\subsection{The objective function}

The optimization criterion is the minimum of the capital costs $\mathrm{C}$ and the expenses $\mathrm{E}$ of the operation of the power supply system for connection of the consumer to the electrical networks

$$
E_{\mathrm{n}} C+E \rightarrow \min
$$

where $E_{n}=0,125$ - normative coefficient of efficiency of investments for electric power industry.

Since the term of the construction of most of the projects for connecting consumers to electrical networks is less than one year, it is permissible to use a simpler criterion - the method of reduced costs instead of the discounted cost method.

The damage was not included in the objective function for the following reasons. Economic sanctions against the power grid company arise only in cases when the factual values of the reliability indices of the enduser power supply $N_{f}$ or $T_{f}$ exceed the contractual $N_{c}$ or $T_{c}$ values [5]. On the other hand, the consumer assesses his risks and chooses the most appropriate level of reliability for him according to the tariff plan. According to the design task, the power supply system must provide reliability indices $N$ and $T$, which should not exceed the contractual values of $N_{c}$ and $T_{c}$. Therefore, the amount of damage will be close to zero and can be neglected to simplify the mathematical model.

Let us consider in detail the components of the objective optimization function.

Capital costs for the construction of a section of high or low voltage line

$$
C_{l i}\left(y_{l i j}, F_{l i j}, L_{l i j}\right)-\sum_{j-1}^{3} y_{l i j} c_{l i j}\left(F_{l i j}\right) \cdot L_{l i j},
$$

where $c_{l i j}$ - the functions of specific capital costs for the construction of a line with uninsulated wires, SIP or CL per $1 \mathrm{~km}, \mathrm{cu} / \mathrm{km}$. line

The cost of operating a section of high or low voltage

$$
E_{l i}\left(y_{l i j}, F_{l i j}, L_{l i j}, P_{\max }\right)-\sum_{j-1}^{3} y_{l i j} e_{l i j}\left(F_{l i j}, P_{\max }\right) \cdot L_{l i j},
$$

where $e_{l i j}$ - the functions of unit operating costs for the construction of a line with uninsulated wires, SIP or CL per $1 \mathrm{~km}$, cu $/ \mathrm{km}$.

Capital costs for a high-voltage or low-voltage power circuit breaker

$$
C_{b i}\left(y_{b i j}, I_{n i j}, I_{s c i j}\right)-\sum_{j-1}^{4} y_{b i j} C_{b i j}\left(I_{n i j}, I_{s c i j}\right),
$$

where $C_{b i j}$ - the functions of capital costs for the installation of air, vacuum, oil or electromagnetic circuit breaker, cu; $I_{n}$ - rated current of the circuit breaker, A; $I_{s c}$ - rated short-circuit breaking current $(\mathrm{SC})$ of the circuit breaker, kA.

The cost of operating a high-voltage or low-voltage circuit breaker

$$
E_{b i}\left(y_{b i j}, I_{n i j}, I_{s c i j}\right)-\sum_{j-1}^{4} y_{b i j} E_{b i j}\left(I_{n i j}, I_{s c i j}\right),
$$

where $E_{b i j}$ - the functions of operating costs for of air, vacuum, oil or electromagnetic circuit breaker, cu.

Capital costs for power transformer

$$
C_{t r}\left(y_{t r j}, S_{n t r j}\right)-\sum_{j-1}^{3} y_{t r j} C_{t r j}\left(S_{n t r j}\right),
$$

where $S_{n t r}$ - rated power of the transformer, kVA; $C_{t r j}$ functions of capital costs for installation of oil, dry or SF6 transformer, cu.

Costs of operating a power transformer

$$
E_{t r}\left(y_{t r j}, S_{n t r j}, P_{\max }\right)-\sum_{j-1}^{3} y_{t r j} E_{t r j}\left(S_{n t r j}, P_{\max }\right),
$$

where $E_{t r j}$ - functions of costs for operation of oil, dry or SF6 transformer, cu.

Thus, the total capital cost for the power supply system

$$
C=C_{b 1}-C_{l 1}+C_{t r}+C_{b 2}+C_{l 2} .
$$

Total costs for the power supply system

$$
E=E_{b 1}-E_{l 1}+E_{t r}-E_{b 2}+E_{l 2} \text {. }
$$

\subsection{Restrictions of the objective function}

1 Restrictions on variables.

1.1 The length of the lines must be positive

$$
L_{U W l i}, L_{S I W l i}, L_{C L l i} \geq 0 \text {. }
$$


1.2 Variable types of lines, transformers and switches - binary variables

$$
\begin{aligned}
& y_{U W l i}, y_{S I W l i}, y_{C L l i} \neq 0,1, \\
& y_{O I L t r}, y_{D R Y t r}, y_{S F 6 t r} \in 0,1, \\
& y_{A I R b i}, y_{V A C b i}, y_{O I L b i}, y_{E M b i} \in 0,1 .
\end{aligned}
$$

2 Only one of the line types, transformers or breakers must be used

$$
\begin{aligned}
& y_{U W l 1}+y_{S I W l 1}+y_{C L l 1}=1, \\
& y_{U W l 2}+y_{S I W l 2}+y_{C L l 2}=1, \\
& y_{A I R b 1}+y_{V A C b 1}+y_{\text {OILb1 } 1}+y_{E M b 1}=1, \\
& y_{A I R b 2}+y_{V A C b 2}+y_{\text {OLLb2 } 2}+y_{E M b 2}=1, \\
& y_{\text {OLLtr }}+y_{D R Y t r}+y_{S F 6 t r}=1 .
\end{aligned}
$$

3 The length of the line must be equal to the specified length

$$
L_{U W l 1}+L_{S I W l 1}+L_{C L l 1}+L_{U W l 2}+L_{S I W l 2}+L_{C L l 2}=L .
$$

4 The loss of voltage in electric-supply system must not exceed a predetermined value

$\sum_{i=1}^{2} \sum_{j=1}^{3} y_{l i j} \Delta U_{l i j}\left(F_{l i j}, L_{l i j}, P_{\max }\right)+\sum_{j-1}^{3} y_{t r j} \Delta U_{t r j}\left(S_{n t r}, P_{\max }\right) \leq \Delta U$,

where $\Delta U_{i j}$ - the voltage loss function on the corresponding element (line or transformer), $\%$.

5 According to the maximum allowable current in the line

$$
I_{\max l i j}\left(y_{l i j}, F_{l i j}\right) \geq \frac{P_{\max }}{\sqrt{3} \cdot U_{\text {nli }} \cdot \cos \varphi},
$$

where $U_{n l i}$ - the nominal voltage of the high or low voltage line, $\mathrm{kV}$.

6 Restriction on reliability.

6.1 Number of failures. The average number of failures in the power supply system should not exceed the set value

$$
\sum_{i=1}^{2} \sum_{j-1}^{3} y_{l i j} w_{l i j} L_{l i j}+\sum_{j-1}^{3} y_{t r j} \omega_{t r j}+\sum_{i=1}^{2} \sum_{j-1}^{4} y_{b i j} w_{b i j} \leq N,
$$

where $\omega_{i j}$ - the failure rate of the corresponding element (line, transformer or switch), failure / year.

6.2 Recovery time. The average recovery time in the power supply system should not exceed the set value

$$
\frac{1}{N} \sum_{i=1}^{2} \sum_{j-1}^{3} y_{l i j} w_{l i j} L_{l i j} t_{l i j}+\sum_{j-1}^{3} y_{t r j} w_{t r j} t_{t r j}+\sum_{i=1}^{2} \sum_{j-1}^{4} y_{b i j} w_{b i j} b_{b i j} ; \leq T,
$$

where $t_{i j}$ - the recovery time of the corresponding element (line, transformer or breaker), hour.

7 Power loss in the power supply system must not exceed the set value

$$
\sum_{i=1}^{2} \sum_{j-1}^{3} y_{l i j} \Delta P_{l i j}\left(F_{l i j}, L_{l i j}, P_{\max }\right)-\sum_{j-1}^{3} y_{t r j} \Delta P_{t r j}\left(S_{n t r}, P_{\max }\right) \leq \Delta P,(18)
$$

where $\Delta P_{i j}$ - the power loss function on the corresponding element (line or transformer), $\%$.

8 For breaking capacity.

8.1 The rated breaking current of the circuit breaker must exceed the maximum current

$$
I_{n b}\left(y_{b i j}\right) \geq \frac{P_{\max }}{\sqrt{3} \cdot U_{n i} \cdot \cos \varphi} .
$$

8.2 The rated short-circuit breaking current of the circuit breaker must exceed the short-circuit current

$$
I_{S C b}\left(y_{b i j}\right) \geq I_{P 0 i},
$$

where $I_{S C b}$ the maximum value of the switched-off short circuit current, kA; $I_{P 0}$ the initial value of the periodic component of the short circuit, $\mathrm{kA}$.

8.3 The breaker must disable the aperiodic component of the short-circuit current

$$
i_{a \tau j} \leq i_{a . n o r j},
$$

where $i_{a \tau}$ the estimated value of aperiodic current component of the three-phase short-circuit in circuit breaker in time of short-circuit $\tau$, defined by the expression

$$
i_{a . \tau}=\sqrt{2} I_{P 0} e^{-\frac{\tau}{T_{a}}} ;
$$

$i_{\text {a.nor }}$ normalized value of the aperiodic component in the switch-off current, determined by the expression

$$
i_{\text {a.nor }}=\frac{\sqrt{2} \beta I_{S C b .}}{100},
$$

where $\beta$ content of the aperiodic component in the switch-off current, $\%$.

8.4 According to the enabling capacity, the test shall be carried out according to the condition

$$
I_{P 0 j} \leq I_{i n c j} ; i_{s j} \leq i_{i n c j},
$$

where $I_{\text {inc }}$ rated current of the breaker (the current value of the periodic component); $i_{s}$ shock current of short circuit in the circuit of locates; $i_{\text {inc }}$ the highest peak current of inclusion in the catalog.

The shock current is found by the formula

$$
i_{s}=\sqrt{2} k_{S} I_{P 0},
$$

where $k_{s}$ impact coefficient.

8.5 The electrodynamic resistance of the switch is checked to limit the through current of short-circuit

$$
I_{P 0 j} \leq I_{d y n j} ; i_{s j} \leq i_{d y n j},
$$

where $i_{d y n}$ the highest peak (the rated peak withstand current) in the catalog, kA; $I_{d y n}$ the current value of the periodic component of the limit through-fault current, kA.

8.6 For thermal short-circuit resistance, the switch is checked by condition

$$
B_{K}=I_{P 0 j}^{2} t_{\text {discon.bj }}+T_{a j} \geq I_{\text {ther.bj }}^{2} t_{\text {ther. } b j},
$$

where $t_{\text {discon. } b}$ full-time short-circuit switch c; $T_{a}$ time constant of the aperiodic component of the fault current, c; $I_{\text {ther.b }}$ pre-sensible thermal current circuit breaker, kA; $t_{\text {ther. } b}$ duration of the current thermal resistance, $\mathrm{s}$.

\section{Getting the solution}

The mathematical model allows for the input data to determine:

- Type high and low voltage line can be undertaken, from bare wire, SIW or CL;

- The length of the line of high and low voltage;

- Wire section of each line;

- Type power transformers (oil, dry, SF6);

- Power of the power transformer;

- Type of circuit breaker (air, vacuum, oil and electromagnetic) and brand. 
The algorithm for obtaining a solution for the mathematical model for the rational power supply system is as follows:

1. Enter the initial data;

2. Calculate functions that depend on the source data;

3. We present a mathematical model to the standard form;

4. We solve the problem by one of the methods of nonlinear programming;

5. We analyze the obtained solution.

\section{Conclusion}

The article for the first time developed a mathematical model and optimization algorithm of the power supply system to connect the customer to the power grid according to the scheme of the III category of reliability. The obtained on the basis of mathematical model, rational schemes of power supply can be used by the electric grid organizations for connection of final consumers to electric networks.

\section{References}

1. Vorotnitsky V. V. Reliability of power supply as a tool of regulation of relations between suppliers and consumers of energy // Energy and Management -2009. №3 - pp. 11-15.

2. Oseichuk, V. A. Reliability of supply in a market economy // news electrical engineering: information publishing / founder of ZAO "news elektrotekhnicks". SPb., 2011. - № 1. - pp. 20-22.

3. Oseichuk, V. A. Nepomniaschy V. A. Regulation of reliability and quality of electricity supply // Innovatika and expert-2016. - Issue 1 (16) - pp.175-185.

4. Rules of electrical installation (PUE). 7-e Izd. - M.: NC ENAS, 2004. -222 p.

5. Tereshko O. A. System of economic contract responsibility for the reliability of electricity supply to end consumers//Energoekspert. - Moscow, 2014, № 05. - pp. $12-17$

6. Fedotova G., Voropay N. Optimization of reliability of power supply of consumers // RT\&A. 2007. №2 (6).

URL:

https://cyberleninka.ru/article/n/optimizatsiya-

nadezhnosti-elektrosnabzheniya-potrebiteley (дата обращения: 20.07.2018).

7. L. Zemite, J. Gerhards, M. Gorobetz and A. Levchenkov, "Optimization of distribution system reliability," 2016 IEEE 16th International Conference on Environment and Electrical Engineering (EEEIC), Florence, 2016, pp. 1-6.

8. Ching-Tzong Su and Guor-Rurng Lii, "Reliability optimization design of distribution systems via multilevel hierarchical procedures and generalized reduced gradient method," Energy Management and Power Delivery, 1995. Proceedings of EMPD '95., 1995 International Conference on, 1995, pp. 180-185 vol.1.

9. V. Parada, J. A. Ferland, M. Arias and K. Daniels, "Optimization of electrical distribution feeders using simulated annealing," in IEEE Transactions on Power Delivery, vol. 19, no. 3, pp. 1135-1141, July 2004.

10. R. C. Lotero and J. Contreras, "Distribution System Planning with Reliability," in IEEE Transactions on Power Delivery, vol. 26, no. 4, pp. 2552-2562, Oct. 2011 